\title{
Nuclear Properties of Carbon Nanotubes for Fast Neutron Detection
}

\author{
Jorge Antonio García Gallardo, Sebastián Beneitez Pupo, Mauricio Petaccia and Juana Gervasoni \\ Department of Metallic and Nanostructured Materials (MMyN), Bariloche Atomic Centre (CNEA), San Carlos de Bariloche 8400, \\ Argentina
}

\begin{abstract}
Fast neutron detection is a subject of great relevance in modern nuclear science and engineering, in particular, with the recent advances in nuclear fusion research, detection of fast neutron became a key issue. Nuclear properties of carbon are of special interest due to its relatively high capture cross section for fast neutrons. Devices made of silicon carbide and diamond are based on these properties, and so are being developed to be used with the proper wiring. In addition, in recent years carbon nano-tubes unveiled their electrical and mechanical properties, which can be exploited for neutron detection. In this work, we use MCNP5 Monte Carlo code to analyze the carbon nuclear properties and discuss the way nano-tubes can be used for fast neutron detection.
\end{abstract}

Key words: Fast neutron, nuclear fusion, carbon nano-tube, nano-device.

\section{Introduction}

Fast neutron detectors are of great relevance in nuclear physics, especially in D-T fusion reactors where the spectrum shows a maximum around 14.1 $\mathrm{MeV}$ [1]. The wide ranges of carbon neutron capture properties are usually being exploited in order to detect these neutrons, so new carbon-based technologies are being developed: silicon carbide and diamond.

In the case of silicon carbide ( $\mathrm{SiC})$, the combined effects of both ${ }^{12} \mathrm{C}\left(\mathrm{n}, \mathrm{n}^{\prime}\right){ }^{12} \mathrm{C}$ and ${ }^{28} \mathrm{Si}\left(\mathrm{n}, \mathrm{n}^{\prime}\right)^{28} \mathrm{Si}$ reactions through the ion recoil are the most important channels for neutron detection, unfortunately the sensitivity for these reactions is low, and thin diodes are to be included in the detector which are sensitive to gamma radiation, also present in nuclear reactions [2]. These difficulties are avoided in the case of diamond based detectors; these detectors, which rely on the ${ }^{12} \mathrm{C}(\mathrm{n}, \alpha){ }^{9} \mathrm{Be}$ reaction, are made of a three-layer set in an arrangement very similar to a transistor, by using boron as dopant agent. The $\alpha$ particles and ${ }^{9} \mathrm{Be}$ ions so released to the crystal, do generate

Corresponding author: Jorge Antonio García Gallardo, Dr., investigador, research field: nuclear physics. electron-hole pairs making the whole arrangement work like a semiconducting device [3, 4]. However, the high price of diamond makes this device very expensive for massive implementation.

Due to the continuous improvements in the experimental techniques for synthesizing carbon nano-tubes (CNT) [5], and related nanostructure today is possible to obtain arrangements of vertically aligned nanotubes [6, 7]. This fact makes new nano-devices be designed at least from a theoretical point of view.

In this work we analyse the behaviour of a bulk carbon system of nanotube density, under fast neutron irradiation using MCNP 5, which is a general-purpose transport Monte Carlo N-Particle code that can be used for neutron, photon, electron, or coupled neutron/photon/electron transport, and the way it can be used to detect fast neutron [8].

\section{Discussion}

As can be seen in Fig. 1, we have calculated the reaction rates corresponding to different reaction channels by using the MCNP 5 code. For thermal $(\sim 0.01 \mathrm{eV})$ and epithermal $(1 \mathrm{eV})$ neutrons, just the reaction ${ }^{12} \mathrm{C}(\mathrm{n}, \gamma){ }^{13} \mathrm{C}$ is possible. The most relevant 
channels in the range of fast neutrons (5 -20 MeV) are $(n, n),(n, \gamma),(n, \alpha),(n, p)$ and $(n, d)$ which agreed with previous works [9]. From all these reactions we are considering the first four because the neutron beam energy is about $14.1 \pm 1 \mathrm{MeV}$.

The $(n, \gamma)$ channel yields gamma radiation of three possible energies, depending on the final state of the ${ }^{13} \mathrm{C}$ nucleus. These energies are $4.95 \mathrm{MeV}, 3.68 \mathrm{MeV}$ and $1.26 \mathrm{MeV}$, with probabilities of $67.5 \%, 32.4 \%$ and $0.16 \%$, respectively. Since the final product is also a carbon atom, their chemical properties remain unaltered [10].

The channel $\left(n, n^{\prime}\right)$ is very relevant in the fast neutron range, due to its relatively high reaction rate for $\mathrm{E}>5 \mathrm{MeV}$ and, as it does not produce any transmutation in the material, and has the advantage of not changing the detector composition. The neutron scattered both elastic and inelastic, can be detected indirectly when the carbon ion recoils create electron-hole pairs, being critical thus the conducting properties of the arrangement $[11,12]$.

Despite the (n,n') reaction, the most suitable channels for neutron detection in the range of interest (fast neutrons) are the (n, $\alpha)$ and (n,p), which yields ${ }^{9} \mathrm{Be}$ and ${ }^{12} \mathrm{~B}$, respectively.

Independently of the reaction taking place, the basis of neutron detection is either detecting the outgoing ions, or the changes in the material structure. In the first case electron-hole pairs created inside the arrangement can be detected by a temporal change in the electronic properties of the material, in the second case the changes in the material are expected to be permanent, and are to be considered also as radiation damage. Anyway, detection depends on the electric properties of the material. About this, graphene and carbon nanotubes are particularly interesting, because they exhibit different metallic and semiconducting properties according to its dimension an topology [13, 14]; and also, the presence of impurities and defects in the nanotube structure alters its electrical conductivity, and can be a way of detection $[15,16]$.

In Fig. 2, we show a schematic view of a possible arrangement for dosimetry, where the nanotubes are aligned vertically and parallel each other, with a proper wiring at their ends. This kind of arrangement was already experimentally obtained [7].

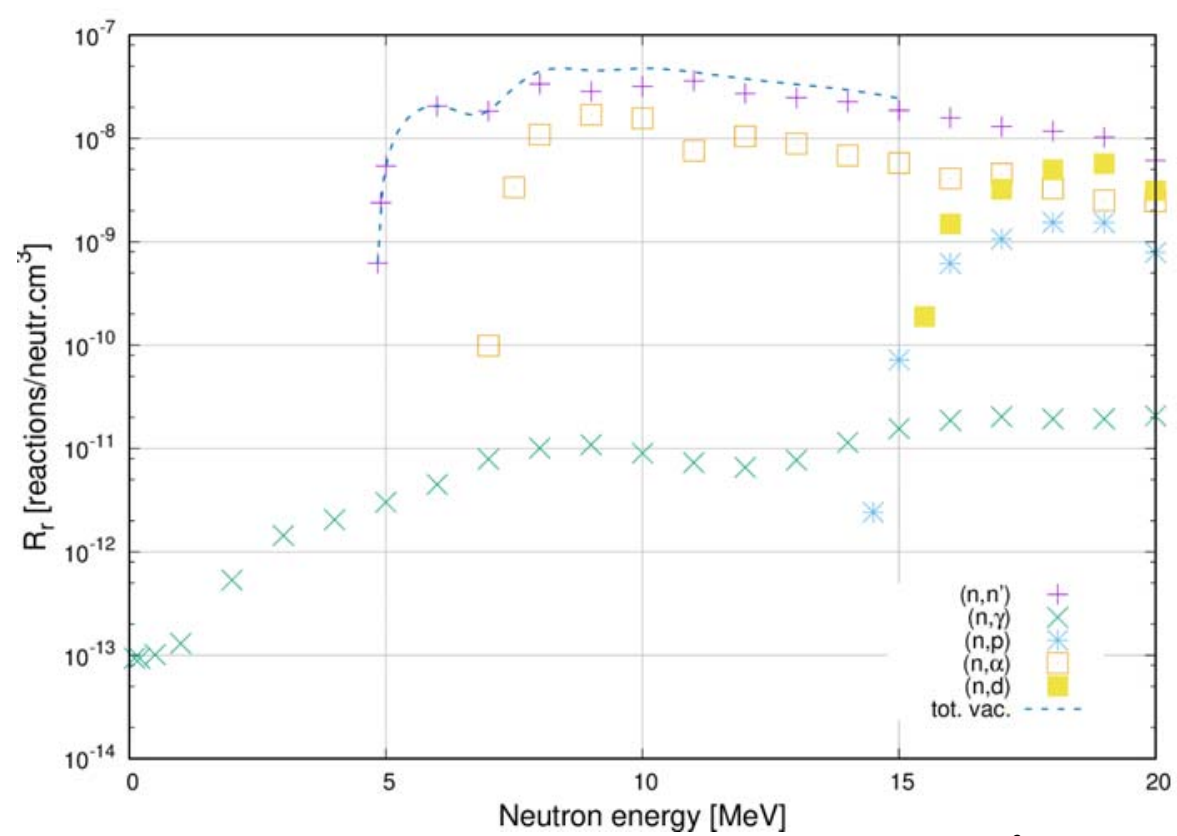

Fig. 1 Normalized reaction rates $R_{r}$ (in reactions over each incident neutron $\times \mathbf{c m}^{3}$ ) for different channels in a nanotube-density carbon array; for energies up to $20 \mathrm{MeV}$, obtained by MCNP5 and database libraries ENDF/B-VI.

Note that the only channel with no threshold is $(\mathrm{n}, \gamma)$. The total generated vacancies (dashed line) in the range $5-15 \mathrm{MeV}$ is also shown. 

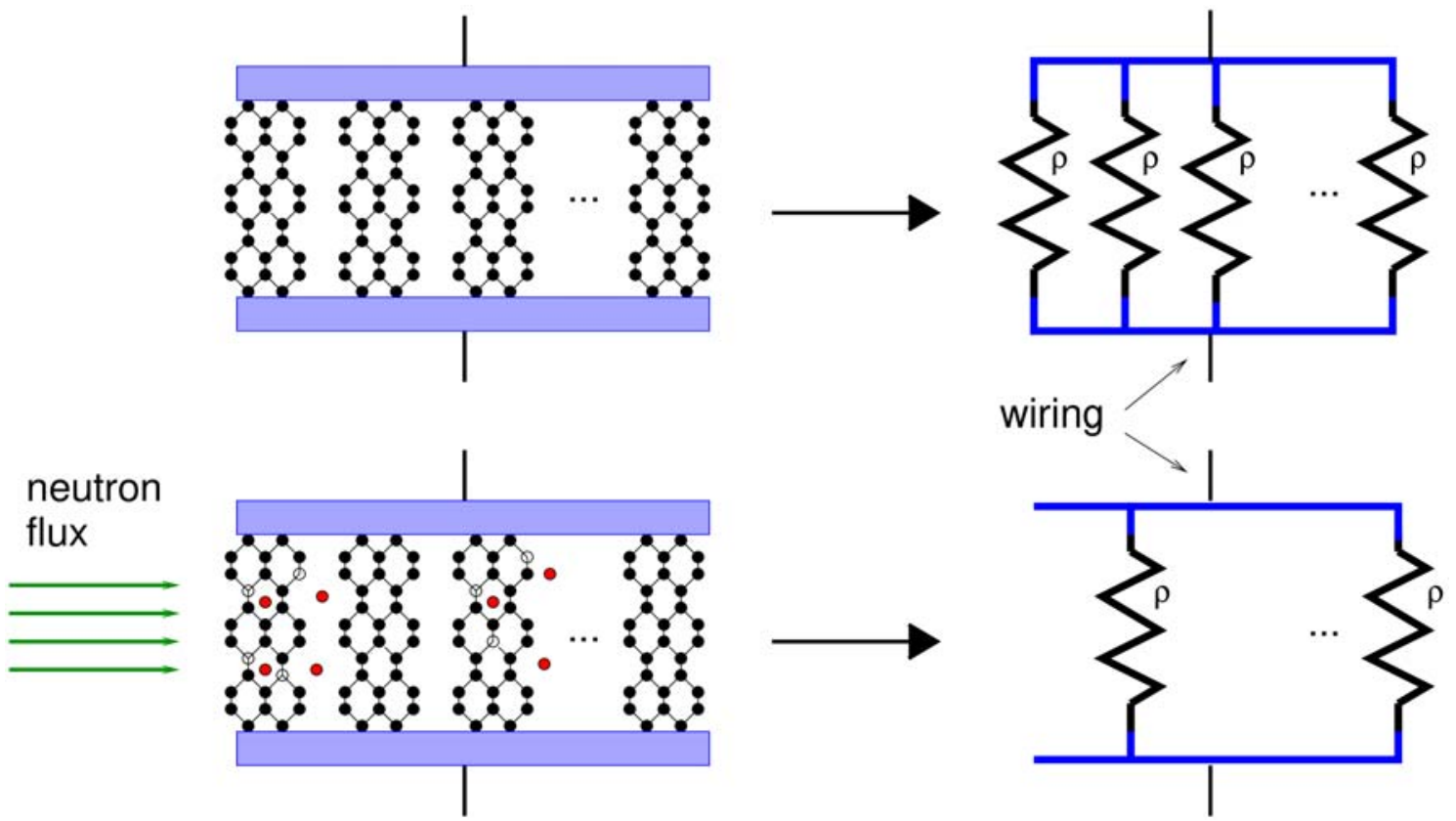

Fig. 2 Scheme of possible dosimeter based on the nanotube change of resistivity.

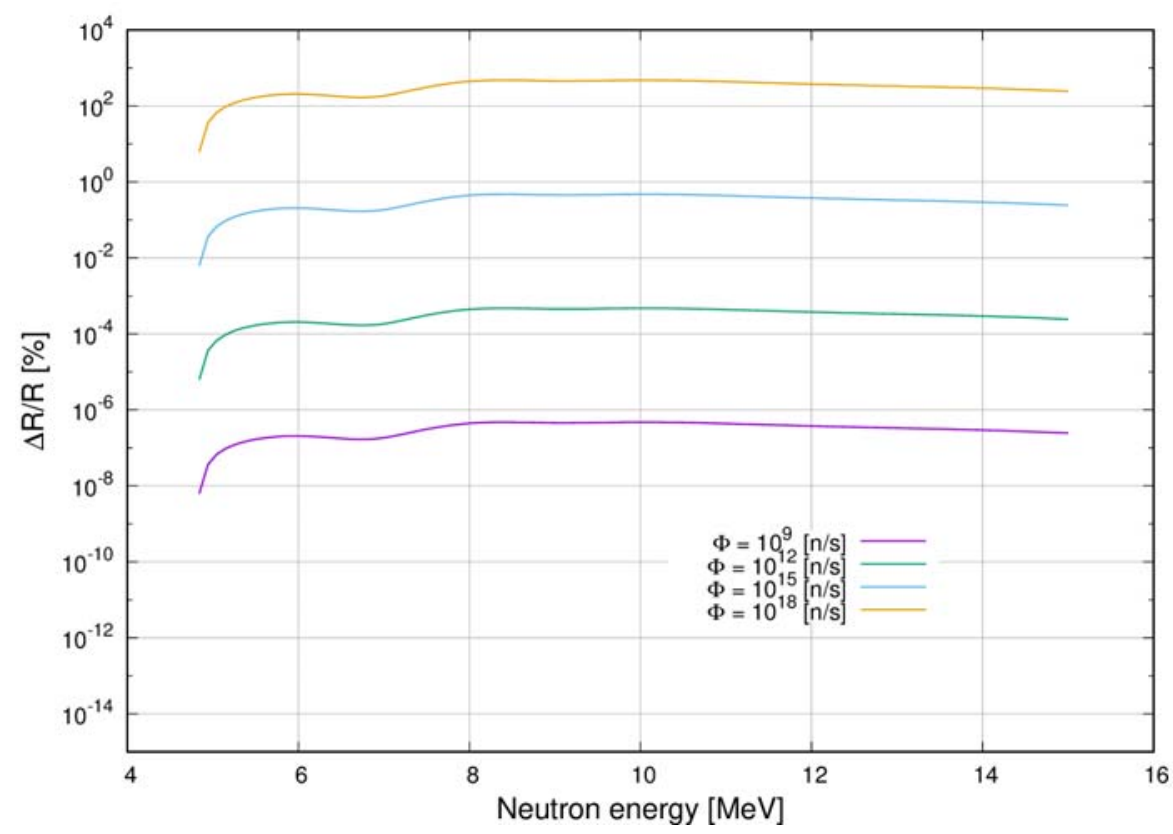

Fig. 3 Relative resistance change in a second as function of incident neutron energy for various neutron fluxes, for an $1 \mathrm{~cm}$ $\times 1 \mathrm{~cm} \times 1 \mathrm{~cm}$ cube detector made of $\left(10^{10}\right.$ nanotubes) SWCNT aligned with the faces.

When a neutron flux traverses the array, the aforementioned nuclear reactions take place, and as the freed energy in this process is relatively high, vacancies in the nanotube are produced, changing so, the electrical properties of the nanotubes. If the nanotubes are single walled (SWCNT) conductivity can even vanish if the nanotube diameter is smaller enough [15]. In this way the system works like an array of parallel resistors, showing at the beginning of the operation a total electric resistance $R=\left[\Sigma^{1} / \rho_{\mathrm{G}_{\mathrm{G}}}\right]^{-1}$ where $\rho$ is the electric resistivity and $G$ is a geometric factor (length over area, for instance), then as the nanotubes are supposed to be equal, we can write the resistance change $\Delta R$ : 


$$
\Delta R / R=\Delta n / n
$$

where $n$ is the number of nanotubes in the array. According to this, for sensitivity reasons, it is better to have a few quantity of long nanotubes than having many-but short of them. Fortunately, this is not a problem because long nanotubes have been successfully obtained, even one half meter long [17].

Fig. 3 shows the variation of relative resistance of an array of $1 \mathrm{~cm}^{3}$, containing $10^{10}$ nanotubes (see Ref. [6]) in a second of irradiation for different neutron fluxes. Varying nanotube density and geometry of the array these values can conveniently be adjusted.

\section{Conclusions}

In this work we used MCNP5 to assess the reaction rates of a bulk made of carbon nano-tubes under fast neutron irradiation. The most interesting channels for neutron detection are $\left(n, n^{\prime}\right),(n, \gamma),(n, \alpha)$ due to their high yield in the range of fast neutron (up to $20 \mathrm{MeV}$ ). Whatever the reaction, due to the high energy released, a vacancy is created, then the ion recoil is able to generate electron-hole pairs. Also, the vacancies in nanotube, change drastically its conducting properties, even more if they are single walled nanotubes (SWCNT). We presented a possible arrangement of such nanotubes in which the permanent damage due to neutron flux, can be recorded in order to determine dose. Such device is technologically possible and its relative resistance $\Delta R / R$ depends on the number of nanotube density and geometry, and can be modified according to flux requirements.

\section{Acknowledgements}

This work was supported by the CNEA (National Commission of Atomic Energy) of Argentina in the frame of the CNEA Controlled Nuclear Fusion Program.

\section{References}

[1] Ericsson, G., Conroy, S., Johnson, M. G., Sundén, E. A., Cecconello, M., Eriksson, J., and JET EFDA Contributors. 2010. "Neutron Spectroscopy as a Fuel Ion Ratio
Diagnostic: Lessons from JET and Prospects for ITER." Review of Scientific Instruments 81 (10): 10D324.

[2] Flammang, R. W., Seidel, J. G., and Ruddy, F. H. 2007. "Fast Neutron Detection with Silicon Carbide Semiconductor Radiation Detectors." Nuclear Instruments and Methods in Physics Research A 579: 177-9.

[3] Almaviva, S., Marinelli, M., Milani, E., Prestopino, G., Tucciarone, A., Verona, C., et al. 2008. "Thermal and Fast Neutron Detection in Chemical Vapor Deposition Single-Crystal Diamond Detectors." J. App. Physics 103: 054501.

[4] Angelone, M., Pillon, M., Prestopino, G., Marinelli, M., Milan, E., Verona, C., et al. 2011. "Thermal and Fast Neutron Dosimetry Using Artificial Single Crystal Diamond Detectors." Radiation Measurements 46: 1686-9.

[5] Endo, M. 1975. "The Growth Mechanism of Vapor-Grown Carbon Fibers." Ph.D. disertation, Universite d'Orleans, France.

[6] Dai, L. M., Patil, A., Gong, X. Y., Guo, Z. X., Liu, L. Q., Liu, Y., et al. 2003. "Aligned Nanotubes." Chem. Phys. Chem. 4 (1): 1150-69.

[7] He, X. W., Gao, W. L., Xie, L. J., Li, B., Zhang, Q., Lei, S. D., et al. 2016. "Wafer-Scale Monodomain Films of Spontaneously Aligned Single-Walled Carbon Nanotubes." Nature Nanotechnology 11: 633-8.

[8] MCNP Version 5, Lawrence J. Cox et al. Los Alamos National Laboratory, LA-UR-02-1527.

[9] Sun, X.-J., Duan, J.-F., Wang J.-M., and Zhang, J.-S. 2007. "Analysis of Neutron Double-Differential Cross Sections for $\mathrm{n}+{ }^{12} \mathrm{C}$ Reaction Below $30 \mathrm{MeV}$." Commun. Theor. Phys. 48: 534-40.

[10] Spilling, P., Gruppelaar, H., De Vries, H. F., and Spits, A. M. J. 1968. "The Reactions $12 \mathrm{C}(\mathrm{n}, \gamma) 13 \mathrm{C}$ and $19 \mathrm{~F}(\mathrm{n}$, ү)20F." Nuclear Physics A 113 (2): 395-411.

[11] Lo Giudice, A. 2005. "Average Energy Dissipated by Mega-Electron-Volt Hydrogen and Helium Ions Per Electron-Hole Pair Generation in 4H-SiC.” Appl. Phys. Lett. 87: 222105.

[12] Gabor1, N. M., Zhong, Z., Bosnick, K., Park, J., and McEuen, P. L. 2009. "Extremely Efficient Multiple Electron-Hole Pair Generation in Carbon Nanotube Photodiodes." Science 325 (5946): 1367-71.

[13] Jorio, A., Dresselhaus, G., and Dresselhaus, M. 2008. Topics in Applied Physics. Berlin: Springer, 111.

[14] Geim, A. K., and Novoselov, K. S. 2007. "The Rise of Grapheme." Nature Mater. 6: 183-91.

[15] Bandaru, P. R. 2007. "Electrical Properties and Applications of Carbon Nanotube Structures." J. of Nanosci. and Nanotech. 7: 1-29.

[16] Lekawa-Raus, A., Patmore, J., Kurzepa, L., Bulmer, J., 
and Koziol, K. 2014. "Electrical Properties of Carbon Nanotube Based Fibers and Their Future Use in Electrical Wiring." Advanced Functional Materials 24 (24): 3661-82.
[17] Zhang, R. F., Zhang, Y. Y., Zhang, Q., Xie, H. H., Qian, W. Z., and Wei, F. 2013. "Growth of Half-Meter Long Carbon Nanotubes Based on Schulz-Flory Distribution." ACS Nano 7 (7): 6156-61. 